EPV013/\#172 HISTOLOGICAL AND MOLECULAR PARTICULARITIES OF BREAST CANCERS IN TUNISIAN POPULATION

${ }^{1} \mathrm{~A}$ Zribi, ${ }^{1} \mathrm{M}$ Ahmad, 'S Ben Nasr, ${ }^{2} \mathrm{~S}$ Fendri, ${ }^{1} \mathrm{~N}$ Mansouri, ${ }^{2} \mathrm{R}$ Arfaoui, ${ }^{1} \mathrm{I}$ Mejri, ${ }^{2} \mathrm{M}$ Balti, ${ }^{2} \mathrm{~A}$ Haddaoui. 'Oncologymilitary Hospital of Tunis, Oncology, Tunis, Tunisia; ${ }^{2}$ Military Hospital of Tunis, Oncology, Tunis, Tunisia

10.1136/ijgc-2021-IGCS.80

Objectives The aim of our study was to determine the distribution of histological subtypes of breast cancer (BC) in Tunisian population and to study their prognostic and therapeutic features

Methods A retrospective study was conducted between January 2011 and December 2018 in our department. A total of 93 cases of $\mathrm{BC}$, using immunohistochemistry, were classified into 4 major molecular subtypes: luminal A, luminal B, HER2-positive, and triple negative

Results Luminal A (50\%) subtype was the most prevalent, followed by triple negative (20\%), HER2-positive (16\%), and luminal B (14\%). Median age was 45 years.90\% of cases were ductal, Axillary lymph nodes were involved in $43 \%$ of all cases and in $73 \%$ of triple negative BC. SBR 23 was found in $64 \%$ of all cases and in $100 \%$ of luminal B BC. Ki67>20 was observed in 92\%, 71\%, 63\% and 5\% of luminal B, HER2-positive, triple negative and luminal A respectively. The DFS at 5 years was $88 \%$.metastatic relapse was observed in $72 \%$ of all population and in $10 \%$ of triple negative subtype. Visceral metastases were observed in $100 \%$ of triple negative $\mathrm{BC}$ while bone metastases werediagnosed in $60 \%$ of luminal Asubtype.In LuminalA subtype, median DFS and OS were respectively 54.4 and 58.4 months, followed bytriple-negative (50.9 and 56.8 months), then HER2-positive(41.7 and 47.4 months)and finally the worst survivalswere attributed to luminalB subtype $(31.3$ and 37.3 months).

Conclusions Our study demonstrated that luminal B BC were characterized by a poor prognosis probably because of the underestimation oftheir aggressiveness and consequently of the less intensive therapeutic management than necessary.

\section{EPV014/\#231 BODY IMAGE PERCEPTION IN TUNISIAN WOMEN FOLLOWING BREAST CANCER}

${ }^{1} Y$ Berrazaga*, ${ }^{1} \mathrm{H}$ Rachdi, ${ }^{1} \mathrm{~N}$ Mejri, ${ }^{2} \mathrm{M}$ Ferjaoui, ${ }^{1} \mathrm{~N}$ Daoud, ${ }^{1} \mathrm{H}$ Boussen. ${ }^{1}$ Abdrahman Mami hospital medical oncology department Tunisia, Medical Oncology, Ariana, Tunisia; ${ }^{2}$ Maternity and Neonatal Center of Tunis, B, Tunis, Tunisia

\subsection{6/ijgc-2021-IGCS.81}

Objectives Patients with breast cancer often receive invasive treatments, with impact on appearance which can affect body image. We aimed to evaluate the impact of breast cancer and its treatments on the body image of Tunisian patients.

Methods Between February and April 2021, thirty-nine patients with breast cancer answered the 10-item body image scale (BIS) questionnaire. Patients responded to questions about relationships with the partner and entourage and to express opinion about breast reconstruction and appearance care.
Results The median age was 47 years. Fifty-four percent underwent Patey's modified radical mastectomy and 46\% lumpectomy with axillary node dissection. All patients received chemotherapy, 54\% radiotherapy, 61\% endocrine therapy and $46 \%$ Trastuzumab. Lymphedema was reported in 20\% of cases. Twenty-three percent were dissatisfied with the scar's appearance. A change for the worse in partner behavior was reported with $8 \%$ of patients. Forty-one percent described society's pitying looks. Only $8 \%$ wanted to undergo a breast reconstruction surgery. Taking care of body appearance was reported to be 'little' by $38 \%$, 'quite a bit' by $54 \%$ and 'very much' by $8 \%$. Twenty-three percent expressed low Self confidence. The median BIS score was 9.69 [1-26]. Score under 10 was reported in $54 \%$ of cases, between 10 and 20 in $38 \%$ and over 20 in $8 \%$. The median BIS score was higher in the radical surgery group $(p=0.048)$.

Conclusions Body Image perception seemed to be affected by the disease and its treatments especially the radical surgery. A psychological care is needed to reduce this impact.

\section{EPV015/\#266 USE OF FLUORESCENCE FOR SENTINEL LYMPH NODE BIOPSY AFTER NEOADJUVANT CHEMOTHERAPY IN INFLAMMATORY BREAST CANCER}

O Kaabia*, R Bouchahda, M Bibi, S Hidar. Université de Sousse, Faculté de Médecine de Sousse, Gynecology and Obstetrics, Sousse, Tunisia

\subsection{6/ijgc-2021-IGCS.82}

Objectives The efficacy and utility of neoadjuvant chemotherapy (NAC) for Inflammatory breast cancer (IBC) are demonstrated. In node-negative patients after NAC, sentinel lymph node biopsy (SLNB) can be considered but not in IBC because of a low identification rate (IR) and a high false-negative rate (FNR). We aim to evaluate the SLNB identification fluorescence technique in this IBC population.

Methods Between 2015 and 2019, data of all patients with NAC for carcinoma of the breast clinically classified as inflammatory (T4d) and without palpably suspicious nodes who underwent an SLNB during the definitive modified radical mastectomy with axillary clearance were retrospectively reviewed. Under general anesthesia $5 \mathrm{ml} / 12.5 \mathrm{mg}$ of Infracyanine ${ }^{\circledR}$ (Indocyanine green) were injected circumferentially around the areola; followed by a $15 \mathrm{~min}$ massage of the breast. The axillary incision was then performed and lymphatic vessels were visualized by a near-infrared camera. The sentinel node(s) were identified as being fluorescent and removed separately. After SLNB biopsy, mastectomy and axillary dissection were fully performed.

Results A total of 22 patients with IBC underwent SLNB after NAC using the above-mentioned technique. The median age at the time of diagnosis was 44 years (range 25-62 years). The identification rate is $86 \%$ (19/22 patients). The false-negative rate was16\% (2 patients among the 12 who had negative SLNB had a positive axilla lymph node examination).

Conclusions The data of this study are encouraging but a larger sample is required. We do not have a clear explanation of why fluorescence provides better IR and lower FNR. 